\title{
UN EXTRAÑO NOMBRE DE LA VENA YUGULAR INTERNA: YUGULAR CIEGA
}

\author{
Juan José Barcia Goyanes \\ Catedrático Jubilado de Anatomía en la Universidad de Valencia
}

\begin{abstract}
RESUMEN
Una errata de la primera obra de Anatomía Humana en persa, Tashrih-i Mansuri, por la que se llama a la yugular interna وداب اعور (widach âçwar), yugular ciega, da lugar al recuerdo de los nombres experimentados por esa vena a lo largo del tiempo y a algunas consideraciones sobre la necesidad de acudir a numerosas fuentes cuando de la identificación de un nombre en los manuscritos se trata.
\end{abstract}

\section{SUMMARY}

A misprint originated in the first work of human anatomy written in Persian, Tashrih-i Mansuri, where the internal jugular ونامج اعور (widach âçwar), is misnamed blind jugular, is the starting point of the remembrance of the names given to this vein throughout the centuries and gives forth some commentaries related with the need to consult many original documents when identifying a name in a manuscript.

Espero que el lector me crea sin necesidad de que se lo jure, si le digo que después de 25 años dedicado al estudio de la historia del lenguaje anatómico me encuentro vacunado frente a cualquier nombre extraño o corrupto de accidentes de nuestro cuerpo. Con todo, no he podido reprimir un movimiento de sorpresa cuando, leyendo la maqala $4^{\mathrm{a}}$, en la que se trata de las venas, de la obra Tashrih-i Mansuri ${ }^{1}$, que estoy traduciendo en la actualidad, me tropecé con un desusado calificativo de la vena yugular interna, yugular ciega.

Como es sabido, las venas yugulares recibieron el nombre con el que todavía las designamos, por su situación en el cuello, jugulum en latín. En realidad tal nombre

1 Tashrih-i Mansuri. Tratado de Anatomía humana en farsi, de fines del siglo XIV o comienzos del $\mathrm{XV}$, compuesto de una muqaddimah, 5 maqalahs y una khatimah. La muqaddimah trata de la definición de los órganos. Las maqalahs, 1. Huesos, 2. Nervios, 3. Músculos, 4. Venas y 5. Arterias. La khatimah, de los miembros compuestos. 
designa propiamente la depresión supraesternal, pero de allí se extendió a la parte anterior del cuello.

El diminutivo de jugum es bastante acertado ya que su situación en el centro del cuello y su prolongación en las dos clavículas recuerda el yugo usado para uncir la pareja de bueyes que arrastraban el arado. Pero de allí se extendió a toda la parte anterior del cuello como se ve, por ejemplo, en las Metamorfosis de Ovidio cuando el poeta, al describir la muerte de Polixena pone en la boca de ésta la frase: "Nula mora est, at tu jugulo vel pectore telum/Conde meo: jugulumque simul pectusque retexit"2. "Huelga la espera: clava tu cuchillo en mi garganta o en mi pecho; y así diciendo descubrió su cuello y su pecho". Se comprende que Polixena no desafía a Pirro a que demuestre su habilidad clavando su daga en la diminuta fosilla supraesternal, apenas visible en las mujeres jóvenes, sino que lo reta a herir su cuello o su pecho, descubriendo ambas regiones.

Pero el adjetivo jugularis no se aplicó a las venas hasta el Renacimiento. Pienso que el primero en hacerlo ha sido Juan Gunterio de Andernach ${ }^{3}$, el médico humanista y anatómico del siglo XV, que fue una de los maestros de Vesalio, el cual (Vesalio) no contó por cierto entre sus virtudes la del agradecimiento a sus maestros. $\mathrm{Y}$ así se cuenta el comentario mordaz que dedicó a Andernach respondiendo a la crítica de Dryander ${ }^{4}$, quien le reprochaba el no haber citado en sus obras a ninguno de sus mentores y, en especial, a Juan Gunterio. Contestaba Vesalio, que "no había querido herir a quien no había visto cortando a hombre ni bruto como no fuera en la mesa", aludiendo así a la escasa afición a la disección de aquél.

Antes del Renacimiento uno de los nombres usados para designar a las yugulares,

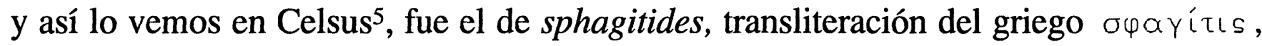
que aparece ya en el Corpus Hipocraticum y que es un derivado del verbo $\sigma \varphi a ́ \gamma \omega$, degollar aplicado a la maniobra con que, en los sacrificios rituales se seccionaban las venas del cuello de la víctima mediante un cuchillo. De ahí se pasó a designar por $\sigma \varphi \propto ү \cap ́$ al propio cuello, como vemos en los grandes centones de nombres griegos de Rufo de Éfeso 6 y Julius Pollux 7 .

Otros de los nombres usados han sido los de vena aperta y vena oculta, aplicados a la jugular externa y a la interna, respectivamente por Constantinus Affer ${ }^{8}$.

2 OVIDIO, Metamorfosis, XIII, 440 y ss.

ANDERNACH, J. G. VON (1554), Institutionum Anatomicarum, 37 b.

4 La observación de referencia se encuentra en la p. 253 de De Radicis Chinae Usus.

5 CELSUS, De Medicina, IV, 9.

6 RUFO DE ÉFESO, De partibus corporis humani. He manejado la edición bilingüe de GULIELMUS CLYNCH. London Typis J. Bettenham, 1726.

7 JULIUS POLLUX (1536), Onomastikón (Texto griego), Basileae.

8 CONSTANINUS AFFER, De Locis in Hominis, C. IV. 


\section{UN EXTRAÑO NOMBRE DE LA VENA YUGULAR INTERNA: YUGULAR CIEGA}

Pero ha habido más, como el de venae organicae usado por la Escuela de Salerno, y que se pretende explicar "quia canendo apparent inflatae", "porque se hinchan al cantar", explicación harto improbable. Pienso, más bien, que han sido llamadas así por su proximidad a la tráquea, que fue llamada organum, no por su forma, sino porque así se llamó a todos los instrumentos de viento y aun todos los usados por los músicos si nos atenemos a lo dicho por San Agustín: "Organa dicuntur omnia instrumenta musicorum" 9 . Y la tráquea era considerada como la productora de la voz y así Alberto Magno ${ }^{10}$ llama a la laringe principium cannae (cannae es la tráquea) de suerte que aquélla queda rebajada a ser una parte de ésta.

Con todo, el nombre habitual de las yugulares durante la Edad Media ha sido el árabe $\tau^{1} \mathrm{~s}$, wida $y$ o alguna de las numerosas transliteraciones de que fue objeto en las traducciones al latín de los textos árabes como guidaz, guidez, guidem, guidegi, guidegis. La transliteración de waw por gu fue constante según Vazquez de Benito ${ }^{11}$, mientras que, según Hyrtt ${ }^{12}$, en las traducciones hechas en España el yim fue constantemente transliterado como z, dando guidaz o guidez, y por la fácil confusión en los manuscritos de la $\mathrm{z}$ con la $\mathrm{m}$ habría aparecido el término guidem.

El nombre árabe fue dado primeramente a la vena yugular externa del caballo, animal del que el conocimiento anatómico llegó a gran altura entre los beduínos si bien no alcanzó la lograda en la anatomía del camello.

Del caballo pasó al hombre y en él, a los nombres señalados se añadieron los correspondientes adjetivos para las yugulares externa e interna, únicas conocidas durante mucho tiempo ya que el nombre de yugular anterior no apareció hasta el siglo pasado con Hyrtl ${ }^{13}$.

Y así en los escritos árabes encontramos los nombres de ودأج الذاهر, widaýalzâhar, yugular visible, para la externa y ودأج الغاءر, wida ýal-gaçir, yugular oculta para la interna, nombres que encontramos en Razes, Haly Abbas y Avicena, por citar únicamente los más destacados de los anatómicos que escribieron en esa lengua, aunque eran persas.

Volvamos ahora al extraño nombre al que en el comienzo de este trabajo me refería. Como he dicho aparecía en la maqala $4^{\circ}$ del tratado Tashrih-i Mansuri, obra que pasa por ser el primer tratado de anatomía en lengua farsi y aparecido en el tiempo a caballo de los siglos XIV y XV. Fue su autor Mansur ben Muhammad ben Ahmad

\footnotetext{
9 SAN AguSTín, Enarraciones sobre los Salmos. Edición bilingüe, tomo II, 56, 16. En Obras Completas de San Agustín. Tomo XX, BAC.

10 SAn Alberto Magno (1651), Beati Alberti Magni Operum. Lugduni. Claudi Prost, Li. XIII, Tr. 1, c. 1.

11 VÁzquez de Benito, C.; Herrera, M. T. (1983), Problemas en la transmisión de arabismos. Al Qantara. Vol. IV, fasc. 1 y 2. Madrid.

12 HYRTL, J. (1880), Das Arabische und Hebräische in der Anatomie, Wien.

13 HYRTL, J. (1846), Lehrbuch der Anatomie des Menschen, Wien.
} 
ben Yusuf Faqih Ilyas, fallecido en 1422. Su obra apareció en 1396 si nos atenemos al testimonio de Cyril Elgood ${ }^{14}$, y fue dedicada a Amirzada Pir Muhammad Bahádur Khán. La obra no llevaba título inicialmente y fue conocida como Tashrih bi alTaswir, "La Anatomía Ilustrada". Aunque, como dije, ha sido considerada como el primer tratado persa de Anatomía Humana, tal vez fue precedida en poco tiempo por el "Compendio de Anatomía" de Abu-ul- Majdal-Bayzavi del que se conserva tan sólo un manuscrito existente en el British Museum y del que poseo un microfilme gracias a la atención de esa corporación.

Pero ya mucho antes, en el Siglo XI había aparecido en farsi un verdadero tratado de Anatomía que constituía el libro primero de la obra Zakhira-i-Khwarazmasháihí o "Tesoro del Shah de Khwarazm" de la que fue autor Ismail ben Hasan ben Muhmmad ben Mahmud ben Ahmad al-Husayni, conocido, con no pequeña ventaja en orden a la brevedad, por al-Yuryaní, por ser natural de Yuryán, la ciudad que hoy se llama Gorgan o Gurgan, y que está cerca del río del mismo nombre y de la orilla oriental del mar Caspio y que, habiendo pertenecido en algún tiempo al imperio de los Sasánidas forma parte hoy de Rusia. El libro está dedicado al Shah de Khwarazm que bajo los sasánidas formaba un pequeño estado tributario de aquel imperio, y que está situado al Sur del Mar de Araal. La obra tuvo la misma fama que las de Razes, Haly Abbas o Avicena pero no fue conocida en Occidente al no haber sido traducida al latín. Yo poseo de ella una copia del manuscrito existente en la Bodleian Library con la cifra Per 801, y la magnífica edición impresa en Teherán en 1957 bajo la dirección de Saidi Sirjaní.

El manuscrito cuya copia leía es el WMS Per $233^{15}$ del Instituto Wellcome y la palabra aludida aparecía en un párrafo que reproduzco

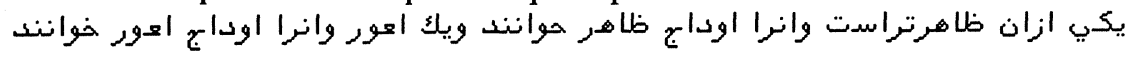

"Una de ellas (de las yugulares) es la más visible y por ello se la llama widach visible y una es ciega y por ello se la llama widach ciega"

Traduzco por "ciega" la palabra اعور, açwâr, que propiamente significa "tuerto". Lo mismo se ha hecho en todas las traducciones que conozco del adjetivo árabe en los dos términos anatómicos en que aparece: el intestino ciego y el introitus canalis nervi facialis $N A$. Y es que tales órganos fueron conocidos por los griegos antes que por los árabes y aquéllos emplearon el adjetivo $\tau \cup \varphi \lambda o ́ s$, que además de ciego, carente de visión, se aplica a un callejón sin salida, por tal tomaron al intestino ciego. $\mathrm{Al}$ ser traducido el término al latín, Celsus ${ }^{16}$ empleó el adjetivo caecum o coecum, pensando también en un callejón sin salida. Por el contrario los árabes, desconocien-

\footnotetext{
14 Elgood, C. (1979), Medical History of Persia. APA-Philo Press. Amsterdam.

15 WMS Per 233. 50 folios, 18 líneas. Naskh de buena mano profesional. Fechado en 1693. 6 dibujos anatómicos.

16 CELSUS, De medicina, IV, 9.
} 
do tal vez esta acepción o juzgándola inaplicable al caso, pensaron que llamar ciego a un tubo que tenía al menos una salida era inadecuado y hablaron de tuerto, lo cual no fue seguido por los traductores de sus obras que utilizaron la expresión grecolatina.

Ya dejo dicho que el calificativo de vena tuerta o ciega, que rompía con la línea clásica de denominaciones de las yugulares me produjo una gran sorpresa. Y lo primero que pensé fue en un error de copista. Compulsé, pues, otro de los Mss. del Tashrif de cuya copia dispongo, el 801 de la Bodleian Library ${ }^{17}$ y en el folio $645 \mathrm{~A}$ encontré la frase reproducida exactamente. No contento con ello busqué la frase en los Mss.Per 221, de la Bodleian Library ${ }^{18}$, Per $612^{19}$ y Per 613 B $^{20}$ del Wellcome Institute y en los tres aparecía la frase, pero la palabra اغور aparecía con un punto diacrítico encima de la çain, o sea que se trataba de ghain y con ello el calificativo de ciega se convertía en "profunda" u "oculta", nombre que concordaba con los calificativos que ya hemos visto eran los habituales en los escritos árabes para la yugular interna.

Y para apurar más mi inquisición consulté la obra ya mencionada de Yuryaní y allí se dice que de las venas es إندرونتر, , "ândaruntar, más profunda" y por ello se llama وداطج باطن, widach, batn, otro de los calificativos frecuentes para indicar la profundidad.

Una variante aparece en el Khulasat-al Tashrih, un compendio de Anatomía del siglo $\mathrm{XV}^{21}$ : el término عاءر , que propiamente designa algo relacionado con el globo ocular por lo que la yugular interna se llamaría "yugular ocular", tal vez porque de ella procede la vena oftálmica. Pero lo más probable es que se trate de una errata por غاء con el sigificado de "oculta" del que ya nos hemos ocupado más arriba.

Por último, y para terminar con nuestra pesquisa por los escritos persas, diremos que en la obra Dashtur-al-fasd ${ }^{22}$ "Tratado de la sangría" en el que se estudian todas las venas conocidas entonces, aparecen las yugulares sin ningún adjetivo.

Para resumir lo dicho podríamos llegar a una conclusión no demasiado novedosa. La de la necesidad de compulsar todas las fuentes a nuestro alcance cuando de manuscritos se trata. De haberme contentado con los dos primeros que cotejé quedaría mi pretensión de haber encontrado un calificativo extraño de la yugular interna y

\footnotetext{
17 BODLEIAN LibraRy MS. PER 801. $2^{\circ}$ Item. En el primer item se recoge la obra de YURYANí, citada en el texto.

18 BODLEIAN LIBRARY MS. WALKER 221, 52 folios. Nasta'liq, buena mano profesional.

19 Ms. WMS Per 612. 19 folios, Naskh de mano profesional. Siglo XVII.

20 Ms. WMS Per 613 B. 28 folios. Seis dibujos anatómicos.

21 Khulasat Al-TASHRih. He manejado el Ms.Wms Per 200 B, del Wellcome Institute. 27 folios, Sikastah.

22 Dashtur AL-FASD. He utilizado el Ms. WMS Per 200 B, del Wellcome Institute. 27 folios. Si-
} kastah. 


\section{JUAN JOSÉ BARCIA GOYANES}

quizás una hipótesis levantada sobre una falsa base para explicar su aparición. Esto les ha ocurrido, por ejemplo a los anatómicos que pretendieron justificar el nombre de vena ranina, nacido, como es sabido de un error de lectura de Gerardo de Cremona ${ }^{23}$.

23 Vide BARCIA GoYANES, J. J. (1990), Onomatología Anatómica Nova, Vol. IX, Suplemento, p. 370 , Valencia. 